\title{
Label-free discrimination of cells undergoing apoptosis by hyperspectral confocual reflectance imaging
}

\section{F. R. Bertani}

\section{E. Botti}

\section{A. Costanzo}

\section{Ferrari}

\section{Mussi}

\section{D'Alessandro}

\section{S. Selci}

Stefano.Selci@isc.cnr.it
CNR-ISC Via del Fosso del Cavaliere 100, 00133 Roma, Italy

Dermatology Department, University of Tor Vergata, Viale Oxford 81, 00133 Roma, Italy

Dermatology Unit - NESMOS Department, Sapienza University of Rome, Italy

CNR-ISC Via del Fosso del Cavaliere 100, 00133 Roma, Italy

CNR-ISC Via del Fosso del Cavaliere 100, 00133 Roma, Italy

CNR-ISC Via del Fosso del Cavaliere 100, 00133 Roma, Italy

CNR-ISC Via del Fosso del Cavaliere 100, 00133 Roma, Italy

Among the optical techniques used for exploring the properties of cells and tissues, those based on hyperspectral label-free analysis are particularly interesting due to their non-invasive character and their ability to fast collect a huge number of information on the different sample constituents and their spatial distribution. Here we present results obtained with a novel hyperspectral reflectance confocal microscope of label-free discrimination of cells undergoing apoptosis. Our data, analyzed by means of a powerful statistical method, enable to obtain information on the biological status at a single cell level through the local measurement of reflectivity. Furthermore, an optical model of the local dielectric response gives an additional insight of the parameters linking the optical responsivity to the biological status. [DOI: http://dx.doi.org/10.2971/jeos.2013.13078]

Keywords: Confocal microscopy, hyperspectral imaging, spectroscopy, cell apoptosis, statistical methods

\section{INTRODUCTION}

One of the main challenges for researchers working in the field of technological advancements for biomedical applications is the development of non-invasive optical tools for early diagnosis [1], fast screening and therapy monitoring [2, 3]. In this context, spectral imaging appears to be a powerful approach which is starting to become applied to medicine $[4,5]$ after it has been largely exploited in other areas, such as mineralogy [6], remote sensing [7], drugs screening [8] and food qualification [9]. In fact, spectral imaging appears to be successful in distinguishing between tumor and normal tissues during surgery [10], and has been used to study endogenous skin fluorophores [11], or to develop a triage test for cervical neoplasia [12]. However, several and complementary methods are needed to analyze the massive amount of data generated by spectral imaging [13] in order to perform unambiguous identification of specific cell markers and tissue status, or to analyze the local structure and composition of biological samples.

Apoptosis or programmed cell death is a complex and well regulated cellular process that, through precise biochemical steps, eventually leads to cell death [14]. The process implies the activation of a large number of molecular pathways and the appearance of relevant morphological changes in the cells. Its study has a great relevance in fields like degenerative dis- eases [15], cancer [16] and development [17]. Here we present early results of label-free discrimination of cells undergoing apoptosis by hyperspectral imaging obtained with a confocal reflectance microscope.

The microscope used for the present study, entirely designed and developed in our labs and fully described elsewhere [18] is equipped with a supercontinuum laser source working in the visible and near infrared region ranging from $0.5 \mu \mathrm{m}$ to $2.4 \mu \mathrm{m}$ wavelengths. Data are collected in such a way that, for any image, the single pixel contains an entire, continuous reflectance spectrum. Our data demonstrate that it is possible to use spectrally encoded data to distinguish the degenerative cell state even when morphology changes are still not visible by standard optical microscopy.

Data analysis has been performed by statistical methods and by modeling the optical cell response, and shows that the apoptotic degeneration is actually related to subtle changes of the optical parameters, making reflectance analysis an interesting alternative to other methods. 


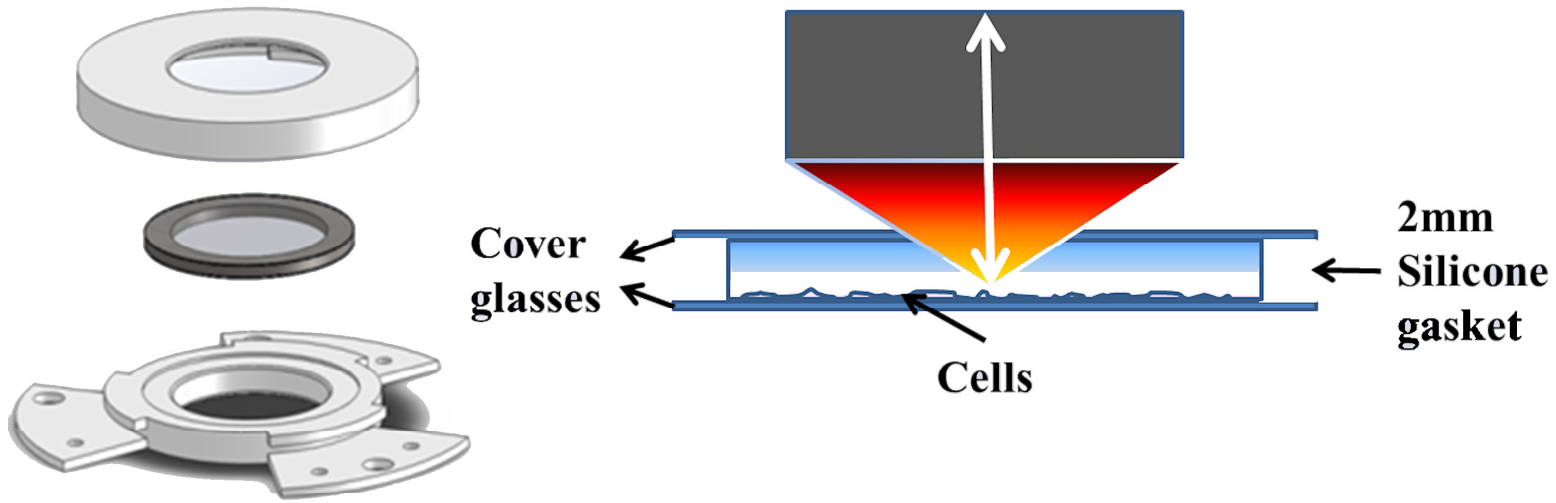

FIG. 1 Assembling scheme of the observation chamber (left panel). Details of the chamber during image acquisition (right panel).

\section{MATERIALS AND METHODS}

We have used HaCaT cells, which are well-known and widely utilized immortalized human keratinocytes. Cells were grown in Dulbecco's Modified Eagle Medium (DMEM) supplemented with $10 \%(\mathrm{v} / \mathrm{v})$ inactivated FBS, $1 \%$ penicillin/streptomycin and 1\% Na Pyruvate.

Cells were plated and cultured on a $40 \mathrm{~mm}$ diameter round coverglass in a $60 \mathrm{~mm}$ Petri dish, and maintained at $37^{\circ} \mathrm{C}$ in a humidified atmosphere of $5 \% \mathrm{CO}_{2} .24$ hours after seeding, cells were exposed to $250 \mathrm{~J} / \mathrm{m}^{2} \mathrm{UVC}$ radiation which represents a common apoptotic stimulus for this cellular model [19]. Exposure has been performed in a Bio-Link BLX 254 crosslinker, at $254 \mathrm{~nm}$ peak wavelength. Negative control cells have been cultured in the same conditions, except for UV exposure. After the exposure, the coverglass was sealed with a previously sterilized $2 \mathrm{~mm}$ thick silicone gasket that holds a second coverglass, and mounted at the base of the observation chamber, sketched in Figure 1, which is fixed on the microscope stage.

In the current setting the microscope is characterized by a lateral resolution of $\lambda / 2$, axial resolution of $4 \mu \mathrm{m}$ and a spectral resolution of $0.8 \mathrm{~nm}$. Images have been acquired over square $500 \times 500 \mu \mathrm{m}$ regions, $256 \times 256$ pixels at $400 \mathrm{~Hz}$ point scan frequency.

Considering laser power emission, focusing optical system characteristics and the culture chamber parameters, we estimated the light power on the cell layer to be less than $4 \mu \mathrm{W} / \mathrm{nm}$. Extensive light exposure has been performed in different cell cultures with no evident damage.

\section{RESULTS AND DISCUSSION}

The evolution of the apoptotic state of HaCaT cells has been investigated during several experiments under the same conditions by the hyperspectral microscope. In each case, the same observed area is imaged at interval of two hours, or less, over at least 24 hours after UVC exposure. The images shown in Figure 2 are related to the $700 \mathrm{~nm}$ wavelength while, as already said, at every image pixel corresponds an entire reflectance spectrum.
Irradiated cells are shown in top panel of Figure 2 at 4 hours, 6 hours and 24 hours after UV exposure. The image acquired at 24 hours clearly shows pyknotic nuclei, which are the consequence of chromatin condensation and the signal of late apoptotic stage, as expected from literature [20]. Images showing unexposed cells (bottom panel in Figure 2) evidence a substantial morphological homogeneity along the observation time. Cells are shown at 2 hours, 6 hours and 24 hours to point out the absence of relevant changes in the whole period.

Within the $500 \mu \mathrm{m} \times 500 \mu \mathrm{m}$ observation field several cells can be identified. In particular, 40 different cells have been selected by placing, for every image, a circular region of interest of about $30 \mu \mathrm{m}$ in diameter inside the cell area and considering the resulting average spectra for analysis purposes. Three time points 4 hours, 6 hours and 24 hours after UV irradiation, corresponding to early, intermediate and late stage of apoptosis process, have been selected to follow the temporal behavior of reflectance spectra at the single cell level. As reflectance reference, we have used a portion of glass substrate free of cells: every spectrum has been normalized to the reference spectrum which takes into account the source, the detector and any other optical spectral response of the apparatus. For each time point, single cellular spectra showed limited variability. To evidence the impact of apoptosis on the optical properties, we averaged the resulting normalized spectra of all the 40 irradiated cells referred to a given time point. The results are plotted in the left side of Figure 3.

We observed a marked difference in the spectral behavior of the cells going ahead in the apoptosis path. On the contrary, the spectra of the control sample, visible in the right side of Figure 3, showed no evidence of analogous changes over the same time interval.

At present, we are not able to define an exact link between optical properties and biochemical state of cell because the relevant changes in spectral profiles are due both to morphological changes and to biochemical modifications. Morphological alterations include cell shrinkage and rounding, nuclear condensation and formation of apoptotic bodies, while biochemical signals include caspase cascade activation and cytochrome $\mathrm{C}$ release from mitochondria. Therefore, we tried to use statistical methods to assess whether there is a correspondence between the optical spectrum of any single cell and its degeneration state during the considered time lapse. The re- 

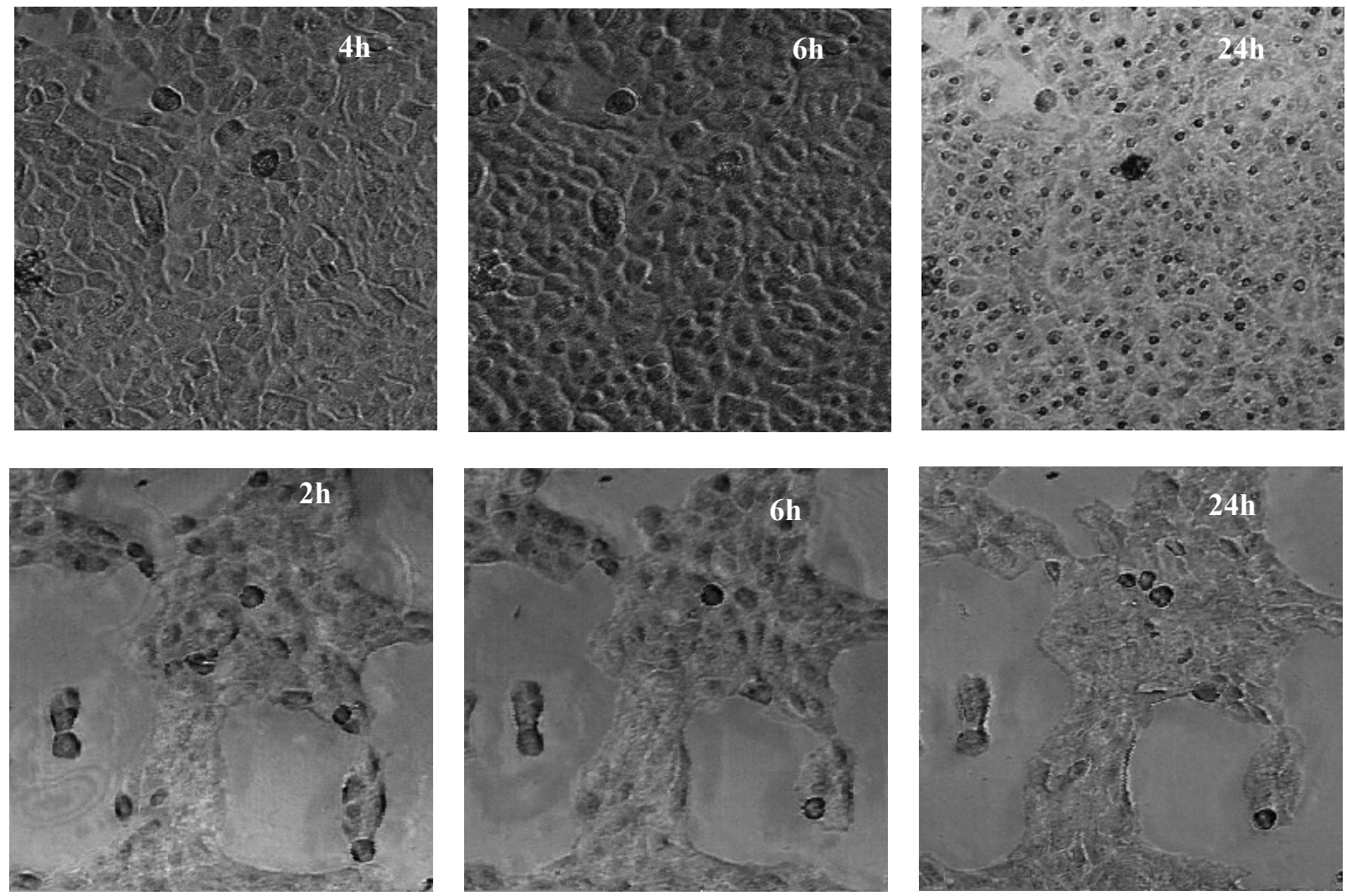

FIG. 2 Confocal images of HaCaT cells in irradiated (top) and not irradiated (bottom) samples. Top panel: from left to right at 4 hours, 6 hours and 24 hours after UV irradiation. Bottom panel: from left to right at 2 hours, 6 hours and 24 hours of observation. All the images are shown for the $700 \mathrm{~nm}$ wavelength. The size of each image is $500 \mu \mathrm{m} \times 500 \mu \mathrm{m}$.
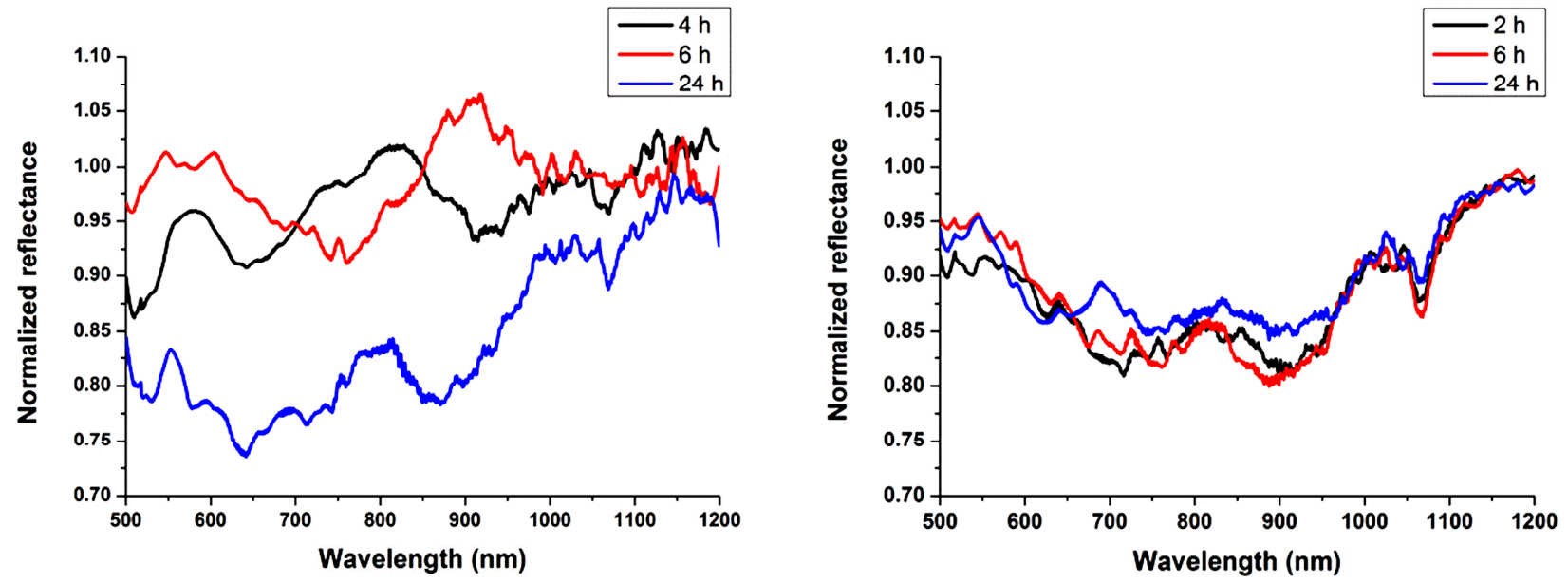

FIG. 3 Reflectance spectra obtained as the average over normalized single cell spectra for each time point. Left side: spectra at 4 hours (black line), 6 hours (red line) and 24 hours (blue line) after exposure. Right side: negative control spectra at 2 hours (black line), 6 hours (red line) and 24 hours (blue line).

flectance spectra from cells at different time points have been considered for Principal Component Analysis (PCA).

PCA is a multivariate statistical analysis technique based on eigenvalue decomposition of the covariance matrix of the hyperspectral image data to be analyzed, and is successfully applied in different fields. It represents a first step of classification and compression of complex hyperspectral dataset in remote sensing [21], food [22], drugs [23], artworks [24] analysis, biomaterials characterization [25], and cell analysis [18]. Averaged and normalized spectra from different cells have been smoothed with a Savitzky-Golay algorithm and compared using PCA, valuating the intensities over 25 discrete wavelengths, spanning from $500 \mathrm{~nm}$ up to $1100 \mathrm{~nm}$, and with a spacing of $\Delta \lambda=25 \mathrm{~nm}$. As it is well known, PCA projects the used intensities in a space where the calculated eigenvectors are considered as new variables. Then, it is possible to visualize data over the two most significant coordinates, usually called PC1 and PC2. Those first two principal components have been taken into account, representing a cumulative variance of $91.35 \%$. Points representing cellular spectra have been plotted in a PC1/PC2 space with markers specific for each time point, as shown in Figure 4. In the score plot of PCA reported in Figure 4, the data acquired after 4 hours are shown in black, after 6 hours in red and after 24 hours in blue. PCA allows a clear discrimination among spectra from different time points. In particular, early and intermediate stages of apoptosis process lie in the PC1 positive half plane, while the late 
stage is in the negative one, indicating a marked change in the spectral pattern. An apparent separation is also present considering PC2: points representing cellular spectra from early stage are in positive half plane, while points representing intermediate stage appear concentrated in the negative one.

The wavelengths assumed as variables for the analysis have been chosen with no a priori knowledge, and different sets of wavelengths has led to consistent results.

Since there is a correspondence between the apoptosis stage of degeneration and the reflectance optical spectrum, within the obvious biological intrinsic variability and degree of statistical uncertainty, we have tried to give a precise meaning to the measured optical spectra. This can be done by modeling the optical response of any single cell as ascribed to a specific thickness $d$ and index of refraction $n_{c}$. Because the optical spectrum considered here is averaged over the entire cell, the thickness and index of refraction are to be considered average effective entities. Moreover, the natural variability associated to any single cell, which is evident in the PCA score plot of Figure 4, makes those values not identical for different cells. However, the analysis can be extremely useful to establish, for instance, the detailed dependence of reflectivity on sensible parameters, such as the refractive index which depends on chemical composition and material density.

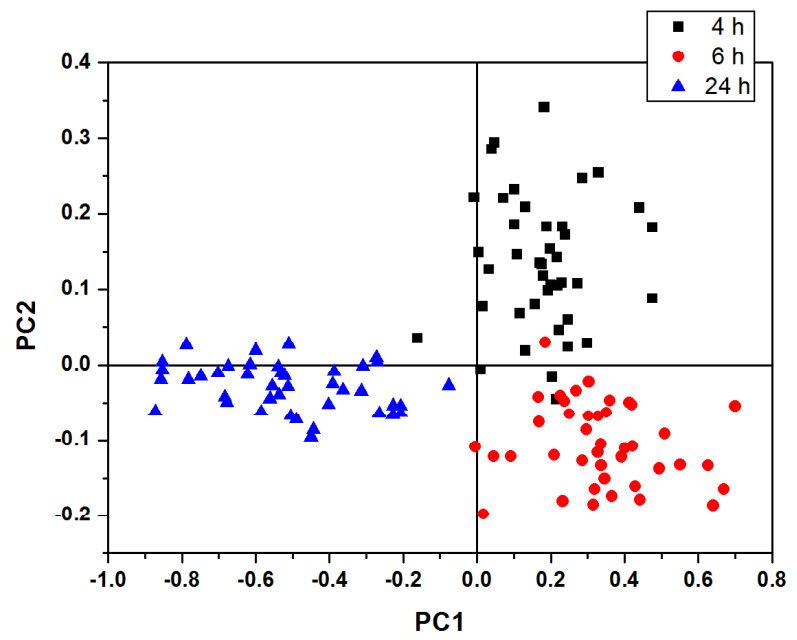

FIG. 4 Score plot of Principal Component Analysis: points represent spectra of the individual cells at different times after UV exposure.

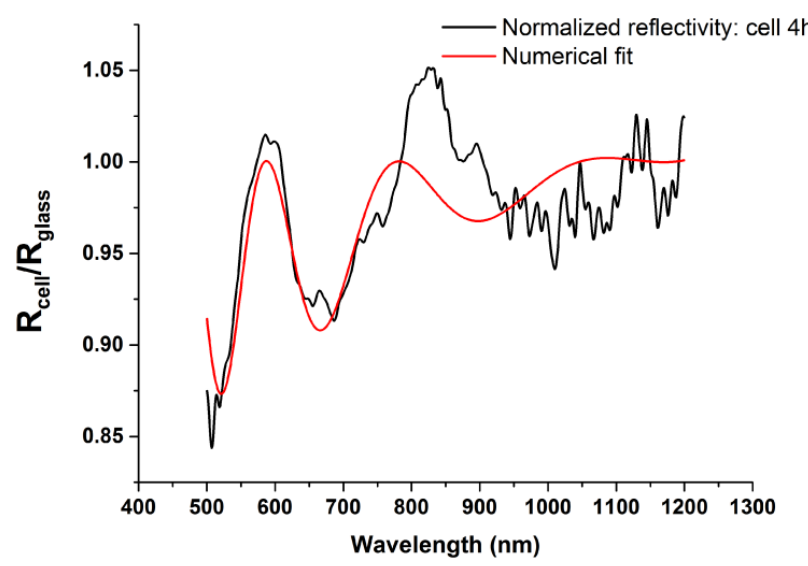

Confocal techniques get the optical reflectivity by limiting the collected information to a close surrounding of the analyzed sample depth (a few microns), so that in the model we can completely disregard the real sequence of intermediate glass layers constituting the observation chamber, and concentrate the attention only to the glass interface around the culture plane. The sample reflectivity is then calculated for a beam irradiating from a semi-infinite zone, the culture medium, impinging over the cells, or over the bare substrate if no cell exists in that point, and being reflected at the glass semi infinite part. The calculation of such a system can be completely carried out in an analytical way, using methods that descend from the Abelés matrix formalism [26]-[29], and have been already used for similar applications [30]. It is possible to write an explicit equation that describes the reflectivity of this simple case.

$$
\left\{\begin{array}{l}
R \cong A\left(n_{1}, n_{2}, n_{c}\right) \cos \left(\frac{2 \pi n_{c} d_{c}}{\lambda}\right)^{2}+C\left(n_{1}, n_{2}, n_{c}\right) \\
A\left(n_{1}, n_{2}, n_{c}\right)=-\frac{\left(n_{c}^{2}-n_{1}^{2}\right)\left(n_{c}^{2}-n_{2}^{2}\right)\left(n_{1}+n_{2}\right)^{2}}{\left(n_{1} n_{2}+n_{c}^{2}\right)^{2}\left(n_{1}-n_{2}\right)^{2}} \\
C\left(n_{1}, n_{2}, n_{c}\right)=\frac{\left(n_{1} n_{2}-n_{c}^{2}\right)^{2}\left(n_{1}+n_{2}\right)^{2}}{\left(n_{1} n_{2}+n_{c}^{2}\right)^{2}\left(n_{1}-n_{2}\right)^{2}}
\end{array}\right.
$$

Note that the sample reflectivity $\mathrm{R}$ has been already normalized to the bare reflectivity of the liquid glass interface. In Eq. (1), $n_{c}$ and $d_{c}$ are the index of refraction and the thickness of the cell model, and $n_{1}$ and $n_{2}$ are the index of refraction of water and glass, respectively. The expression of $R$ has been slightly simplified omitting the term $\left(n_{c}^{2}-n_{1}^{2}\right)\left(n_{c}^{2}-n_{2}^{2}\right) \cos \left(2 \pi n_{c} d_{c} / \lambda\right) \quad$ respect to the term $\left(n_{1} n_{2}+n_{c}^{2}\right)^{2}$ at the denominator. The omission causes a maximum inaccuracy effect of the order of $10^{-3}$, corresponding to the case in which the cosine term is equal to one.

It is possible to use the expression (1) to numerically fit the reflectance of any of the single cells visible in Figure 2. Figure 5 presents the fit results of the reflectance of one cell 4 hours (left), and 6 hours (right), after apoptotic stimulus.

The numerical fit assumes constant values for the refractive index of glass $\left(n_{2}=1.516\right)$ and of culture liquid, considered as simple water $\left(n_{1}=1.33\right)$. This last assumption is only approximate, because it does not consider the many chemical components included in that liquid. Despite these approximations,

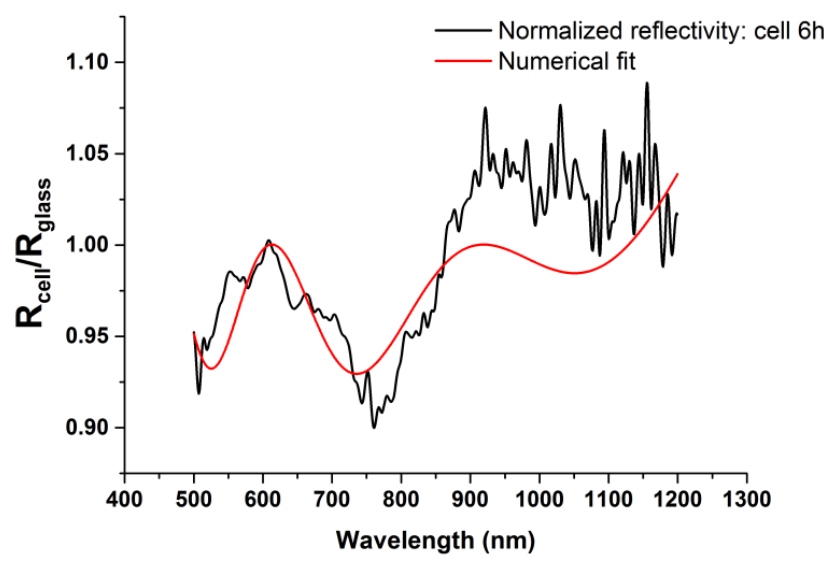

FIG. 5 Experimental reflectance (black line) and numerical fit (red line) after 4 hours, left, and after 6 hours, right, from UV irradiation. 


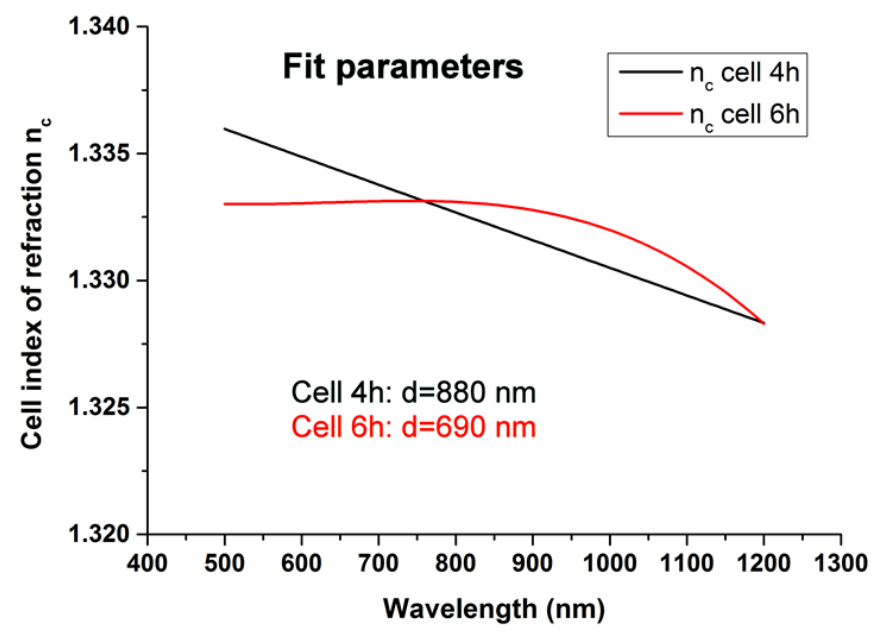

FIG. 6 Fit parameters of one cell after 4 hours and after 6 hours.

the fit is quite accurate in reproducing the general shape of the experimental reflectivity. The fit is able to estimate two main parameters: the cell thickness $d_{c}$, and the cell index of refraction $n_{c}$, including its dispersion. The obtained parameters are visible in Figure 6. The results indicate that between four and six hours after UV irradiation, during the apoptosis process, a small collapse of the cell structure takes place, indicated by the reduction of $d$, which is consistent with the general trend of the cellular degeneration. Moreover, the value and the dispersion of the cell index of refraction slightly changes, indicating a chemical modification that induces a variation of the overall optical properties.

\section{CONCLUSIONS}

We demonstrate that the sensitivity of reflectance to very small alterations of the optical parameters is quite remarkable and makes the presented method interesting for the spectroscopic labeling of biological objects. In particular, we show that it is possible to spectrally distinguish near temporal stages of a complex phenomenon like apoptosis without any exogenous dye and in absence of univocal chemically specific spectral bands. Our results highlight the potentiality of reflectance hyperspectral microscopy in the field of biological research, and represent a step further in the way towards the direct application of this powerful technique in the fields of bio-analytics and diagnostics.

\section{ACKN OWLEDGEMENTS}

We want to thank Barbara Marinari, Francesca Moretti e Valentina Pinetti (Dermatology Dept. University Tor Vergata), for samples culture and preparation. IDEAS-FIRB "Skintarget" grant, contract number RBID08W2TR, AIRC “Investigator Grant" grant to AC.

\section{References}

[1] M. A. Calin, S. V. Parasca, R. Savastru, M. R. Calin, and S. Dontu, "Optical techniques for the noninvasive diagnosis of skin cancer," J. Cancer. Res. Clin. Oncol. 139, 1083-1104 (2013).
[2] N. Bedard, R. A. Schwarz, A. Hu, V. Bhattar, J. Howe, M. D. Williams, A. M. Gillenwater, R. Richards-Kortum, and T. S. Tkaczyk, "Multimodal snapshot spectral imaging for oral cancer diagnostics: a pilot study," Biomed. Opt. Express 4, 938-949 (2013).

[3] J. Q. Brown, T. M. Bydlon, S. A. Kennedy, M. L. Caldwell, J. E. Gallagher, M. Junker, L. C. Wilke, W. T. Barry, J. Geradts, and N. Ramanujam, "Optical spectral surveillance of breast tissue landscapes for detection of residual disease in breast tumor margins," PLOS ONE 8, e69906 (2013).

[4] M. E. Martin, M. B. Wabuyele, K. Chen, P. Kasili, M. Panjehpour, M. Phan, B. Overholt, G. Cunningham, D. Wilson, R. C. Denovo, and T. Vo-Dinh, "Development of an advanced hyperspectral imaging (HSI) system with applications for cancer detection," Ann. Biomed. Eng. 34, 1061-1068 (2006).

[5] A. M. Siddiqi, H. Li, F. Faruque, W. Williams, K. Lai, M. Hughson, S. Bigler, J. Beach, and W. Johnson, "Use of hyperspectral imaging to distinguish normal, precancerous, and cancerous cells," Cancer Cytopathol. 114, 13-21 (2008).

[6] D. C. Heinz, and I. C. Chein, "Fully constrained least squares linear spectral mixture analysis method for material quantification in hyperspectral imagery," IEEE T. Geosci. Remote 39, 529-545 (2001).

[7] A. F. H. Goetz, G. Vane, J. E. Solomon, and B. N. Rock, "Imaging spectrometry for earth remote sensing," Science 228, 1147-1153 (1985).

[8] G. L. Alexandrino, and R. J. Poppi, “NIR imaging spectroscopy for quantification of constituents in polymers thin films loaded with paracetamol," Anal. Chim. Acta 765, 37-44 (2013).

[9] D. Wu, and D.-W. Sun, "Application of visible and near infrared hyperspectral imaging for non-invasively measuring distribution of water-holding capacity in salmon flesh," Talanta 116, 266-276 (2013).

[10] S. V. Panasyuk, S. Yang, D. V. Faller, D. Ngo, R. A. Lew, J. E. Freeman, and A. E. Rogers, "Medical hyperspectral imaging to facilitate residual tumor identification during surgery," Cancer Biol. Ther. 6, 439-446 (2007).

[11] J. Spigulis, "Biophotonic technologies for non-invasive assessment of skin condition and blood microcirculation," Latvian J. Phys. Techn. Sci. 49, 63-80 (2012).

[12] L. B. Twiggs, N. A. Chakhtoura, D. G. Ferris, L. C. Flowers, M. L. Winter, D. R. Sternfeld, M. Lashgari, A. F. Burnett, S. S. Raab, and E. J. Wilkinson, "Multimodal hyperspectroscopy as a triage test for cervical neoplasia: pivotal clinical trial results," Gynecol. Oncol. 130, 147-151 (2013).

[13] L. Görlitz, B. H. Menze, B. M. Kelm, and F. A. Hamprecht, "Processing spectral data," Surf. Interface Anal. 41, 636-644 (2009).

[14] J. F. Kerr, A. H. Wyllie, and A. R. Currie, "Apoptosis: a basic biological phenomenon with wide-ranging implications in tissue kinetics," Br. J. Cancer 26, 239-257 (1972).

[15] M. P. Mattson, "Apoptosis in neurodegenerative disorders," Nat. Rev. Mol. Cell Biol. 1, 120-130 (2000).

[16] S. W. Lowe, and A. W. Lin, "Apoptosis in cancer," Carcinogenesis 21, 485-495 (2000).

[17] P. Meier, A. Finch, and G. Evan, "Apoptosis in development," Nature 407, 796-801 (2000).

[18] F. R. Bertani, L. Ferrari, V. Mussi, E. Botti, A. Costanzo, and S. Selci, "Living matter observations with a novel hyperspectral supercontinuum confocal microscope for VIS to near-IR reflectance spectroscopy," Sensors 13, 14523-14542 (2013). 
[19] U. Henseleit, T. Rosenbach, and G. Kolde, "Induction of apoptosis in human HaCaT keratinocytes," Arch. Dermatol. Res. 288, 676-683 (1996).

[20] R. Takasawa, H. Nakamura, T. Mori, and S. Tanuma, "Differential apoptotic pathways in human keratinocyte $\mathrm{HaCaT}$ cells exposed to UVB and UVC," Apoptosis 10, 1121-1130 (2005).

[21] G. F. Byrne, P. F. Crapper, and K. K. Mayo, "Monitoring land-cover change by principal component analysis of multitemporal landsat data," Remote Sens. Environ. 10, 175-184 (1980).

[22] M. Kamruzzaman, D.-W. Sun, G. ElMasry, and P. Allen, "Fast detection and visualization of minced lamb meat adulteration using NIR hyperspectral imaging and multivariate image analysis," Talanta 103, 130-136 (2013).

[23] R. Carneiro, and R. Poppi, "A quantitative method using near infrared imaging spectroscopy for determination of surface composition of tablet dosage forms: an example of spirolactone tablets," J. Braz. Chem. Soc. 23, 1570-1576 (2012).

[24] G. Sciutto, P. Oliveri, S. Prati, M. Quaranta, S. Bersani, and R. Mazzeo, "An advanced multivariate approach for processing $\mathrm{X}$-ray fluorescence spectral and hyperspectral data from noninvasive in situ analyses on painted surfaces," Anal. Chim. Acta 752, 30-38 (2012).
[25] B. J. Tyler, G. Rayal, and D. G. Castner, "Multivariate analysis strategies for processing ToF-SIMS images of biomaterials," Biomaterials 28, 2412-2423 (2007).

[26] M. Born, and E. Wolf, Principles of Optics: Electromagnetic Theory of Propagation, Interference and Diffraction of Light (Cambridge University Press, Cambridge, 1999).

[27] A. Cricenti, S. Selci, F. Ciccacci, A. C. Felici, C. Coletti, Y. Zhu, and G. Chiarotti, "Determination of the complex dielectric function of $\mathrm{Si}(111) 2 \times 1, \operatorname{GaAs}(110)$ and $\mathrm{GaP}(110)$ surfaces by polarized surface differential reflectivity," Phys. Scripta 38, 199-203 (1988).

[28] S. Nannarone, and S. Selci, "Dielectric properties of the Si(111) $2 \times 1$ surface: Optical constants and the energy-loss spectrum," Phys. Rev. B 28, 5930-5936 (1983).

[29] S. Selci, A. Cricenti, F. Ciccacci, A. C. Felici, C. Coletti, Z. Yong, and G. Chiarotti, "Dielectric functions of $\mathrm{Si}(111) 2 \times 1, \mathrm{Ge}(111) 2 \times 1$, $\operatorname{GaAs}(110)$ and $\operatorname{GaP}(110)$ surfaces obtained by polarized surface differential reflectivity," Surf. Sci. 189, 1023-1027 (1987).

[30] S. Selci, F. R. Bertani, and L. Ferrari, "Supercontinuum ultra wide range confocal microscope for reflectance spectroscopy of living matter and material science surfaces," AIP Advances 1, 032143 (2011). 